\title{
The Symptoms and Impact of Recurrent Focal Segmental Glomerulosclerosis in Kidney Transplant Recipients: A Conceptual Model of the Patient Experience
}

\author{
Marci English • Emily Hawryluk - Robert Krupnick • Mysore S. A. Kumar • \\ Jason Schwartz
}

Received: June 21, 2019 / Published online: October 14, 2019

(c) The Author(s) 2019

\section{ABSTRACT}

Introduction: We qualitatively examined the symptoms and impact of recurrent primary focal segmental glomerulosclerosis (rpFSGS) in kidney transplant recipients, compared with two related FSGS populations, to characterize the experience of patients with rpFSGS.

Methods: A literature review identified 58 articles concerning the experience of patients with pFSGS and/or rpFSGS in three groups: pretransplant pFSGS, post-transplant rpFSGS, or

Enhanced Digital Features To view enhanced digital features for this article go to https://doi.org/10.6084/ m9.figshare.9901358.

Electronic Supplementary Material The online version of this article (https://doi.org/10.1007/s12325019-01110-5) contains supplementary material, which is available to authorized users.

M. English $(\bowtie)$

Medical Specialties, Astellas Pharma Global

Development, Inc., Northbrook, IL, USA

e-mail: marci.english@astellas.com

E. Hawryluk · R. Krupnick

Patient Centered Endpoints, IQVIA, Cambridge,

MA, USA

M. S. A. Kumar

Medical Affairs, Astellas Pharma Global

Development, Inc., Northbrook, IL, USA

J. Schwartz

Global Medical Affairs, Astellas Pharma Global

Development, Inc., Northbrook, IL, USA post-transplant non-recurrent pFSGS. Literature findings were used to construct a preliminary conceptual model incorporating the symptoms and impact of rpFSGS, which was refined on the basis of qualitative interviews with clinicians. Twenty-five patients (rpFSGS: $n=15$; pre-transplant pFSGS: $n=5$; post-transplant non-recurrent pFSGS: $n=5$ ) were interviewed to characterize the experience of patients with rpFSGS and compare it with other FSGS populations, and findings were used to finalize the conceptual model.

Results: The impact of pFSGS/rpFSGS described in the literature was diverse. Treatment-related symptoms, along with anxiety and depression, were considered important features of rpFSGS in addition to the findings from the literature review, according to clinicians. Patient-reported tiredness and swelling were the most common/ disturbing symptoms associated with rpFSGS, while physical activity restrictions and adverse effects on work/social life were considered the most profound impact concepts. The collective disease experience was different for patients with rpFSGS and non-recurrent pFSGS, although psychological impact, including treatment-related anxiety and depression, were common to both groups.

Conclusions: Post-transplant recipients with rpFSGS display a greater symptom burden and experience a more diverse impact than those with non-recurrent pFSGS, highlighting the importance of effective patient monitoring and 
introducing effective treatments for the prevention and management of pFSGS recurrence. Funding: Astellas Pharma Global Development, Inc.

Keywords: Conceptual model; Focal segmental glomerulosclerosis; Kidney transplant; Nephrology; Primary focal segmental glomerulosclerosis; Recurrent focal segmental glomerulosclerosis; Recurrent primary focal segmental glomerulosclerosis

\section{INTRODUCTION}

Focal segmental glomerulosclerosis (FSGS) is a common cause of proteinuria and nephrotic syndrome leading to end-stage renal disease (ESRD). It refers to a group of clinicopathological syndromes characterized by focal and segmental scarring in the glomerulus $[1,2]$. Although several factors are implicated in the development of FSGS, a common cause is podocyte injury [1, 3].

Worldwide, the prevalence of FSGS is increasing, although the absolute incidence and prevalence of the condition remain largely unknown $[4,5]$. A systematic literature review based on studies of the incidence of primary glomerulonephritis in Europe, North and South America, Canada, Australasia, and the Middle East reported an overall incidence rate of 0.8 / 100,000 of the population per year for FSGS [6]. Meanwhile, a United States (US)-based population study showed that rates of FSGS had increased annually by $2.7 / 100,000$ person-years over a 12-year observation period, with FSGS accounting for $38.9 \%$ of all primary glomerulonephropathies [7].

FSGS occurs as either primary (idiopathic) FSGS (pFSGS) or as secondary FSGS (sFSGS). pFSGS represents a major type of glomerulonephritis, accounting for about 4\% of ESRD in the USA $[1,2]$, while sFSGS is associated with non-nephrotic proteinuria and shows less clinical severity [1]. Approximately $30-50 \%$ of pFSGS cases will recur following kidney transplantation [8], although the causal mechanisms of recurrent pFSGS (rpFSGS) remain largely unknown $[4,9]$.
RpFSGS is associated with an increased risk of allograft failure and its management represents a clinical challenge $[4,8,10,11]$. High-dose corticosteroid therapy is used as first-line therapy in patients with pFSGS/rpFSGS with nephrotic-range proteinuria [12]; however, no large-scale randomized controlled trials have been conducted to corroborate their efficacy in this setting $[8,10]$. Although other therapies are emerging, investigations are in their early stages and, to date, FSGS-specific proof-of-concept studies have not yet appeared in the literature.

Although studies have investigated the quality of life (QoL) burden of pFSGS [13-15], very few have explored the impact of disease recurrence on QoL in kidney transplant recipients. Using a three-tiered methodological approach involving a targeted literature review and qualitative interviews with clinicians and patients, this study qualitatively examined the symptoms and impact of rpFSGS in kidney transplant recipients compared with two similar populations (patients with pFSGS in native kidneys and post-transplant recipients with a history of pFSGS without disease recurrence) to characterize the experience of patients with rpFSGS and further distinguish it from other forms of FSGS.

\section{METHODS}

\section{Literature Review}

A targeted literature review search was undertaken between October 4, 2016 and November 14, 2016 using MEDLINE (PubMed), Google/ Google Scholar, and other select databases to identify relevant articles pertaining to the symptoms of pFSGS and/or rpFSGS and the associated impact of these conditions on patients segregated into three predefined groups: those with pre-transplant pFSGS, posttransplant rpFSGS, or post-transplant non-recurrent pFSGS. The overall search strategy consisted of disease-related terms that captured "FSGS", "recurrent FSGS", "early recurrence" or "late recurrence", "proteinuria" or "nephrotic syndrome", "hypoalbuminemia", "hyperlipidemia", "kidney transplant", or "kidney 
Table 1 Literature search strategy and total number of publications identified

Search terms ${ }^{a}$

“recurrent FSGS"

"FSGS"

"proteinuria” or "nephrotic syndrome”

"hypoalbuminemia”

"hyperlipidemia”

“recurrent FSGS”

"FSGS"

"proteinuria” or "nephrotic syndrome"

“recurrent FSGS”

"FSGS"

"proteinuria” or "nephrotic syndrome"

"kidney transplant"

"kidney disease"

"FSGS"

"FSGS"

“recurrent FSGS"

"FSGS"

“proteinuria” or "nephrotic syndrome”

"kidney transplant"

"kidney disease"

"kidney loss"

"FSGS"

“FSGS” vs "recurrent FSGS"

"early recurrence" and "late recurrence" and "FSGS"

“dialysis"

"suicide” and "dialysis"

“immunosuppressant” and "kidney”

“plasmapheresis”

“rituximab”
Database $^{\text {b }}$

and "symptoms"

PubMed

retrieved

76

1550

45,278

3202

32,878

and "clinical manifestations"

PubMed

1

and "patient-reported outcome"

PubMed

\section{0}

0

1

8

19

and "Sanofi"

Google

8810

and "GSK"

Google

117,000

and "quality of life"

PubMed

2096

7174

and "emotional impact" or "economic impact"

PubMed

1241

PubMed 10

PubMed

and "quality of life" or "side

PubMed

3717 effects" 
Table 1 continued

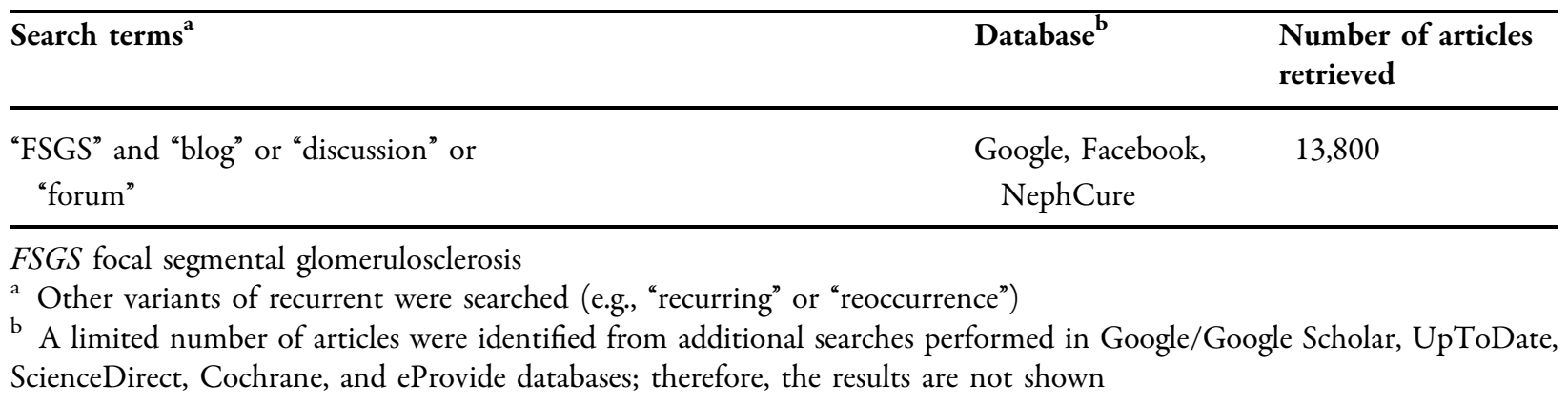

disease"; and terms related to impact including "symptoms", "clinical manifestations", "patient-reported outcome", "quality of life", "emotional impact", or "economic impact" (Table 1). In addition, searches were conducted to identify FSGS/rpFSGS-related articles on disease or kidney transplant-specific, patient-reported outcome (PRO) instruments. Google, Facebook, Twitter, and NephCure Kidney International were also searched to capture blogs or forum discussions relating specifically to patient experience.

To be eligible for inclusion, all articles had to feature one or more of the following concepts: FSGS or rpFSGS; symptoms and impact of rpFSGS or FSGS; signs, symptoms, and impact of kidney disease; QoL in kidney transplant recipients; online posts from patients with FSGS or rpFSGS; PROs pertaining to FSGS, kidney disease, or kidney transplant; treatments for rpFSGS; and the effects of those treatments. Articles were excluded if they did not provide any details on signs, symptoms, and/or impact of rpFSGS, FSGS, or associated conditions; if they concerned prognosis index scores, treatment options (subtype and genetic indicators), classification criteria, or other biological aspects of rpFSGS; or if they were focused on secondary or pediatric ( $<18$ years) cases of FSGS or rpFSGS, respectively. The literature review findings were used to construct a preliminary conceptual model of the signs, symptoms, and impact of rpFSGS, to be refined on the basis of clinician interviews and tested in patient interviews.

\section{Clinician Interviews}

Interviews were conducted to identify important and relevant rpFSGS concepts from a clinician's perspective to refine the preliminary conceptual model. A total of six US physicians, including three transplant nephrologists, one clinical nephrologist, and two transplant surgeons (transplant surgeons were employees of the study sponsor), met the inclusion criteria and were invited by email to participate in a 1-h interview. Clinicians provided verbal consent for their responses to be recorded, and for the recordings to be transcribed and anonymized. A comprehensive discussion guide was designed to obtain information from clinicians regarding healthcare practice in FSGS/rpFSGS. This included questions regarding different patient populations and disease stages (including at diagnosis and pre- versus post-transplant), as well as any differentiating factors of rpFSGS compared with pFSGS (Supplementary Table S1). Symptoms and impact concepts were qualitatively compared to generate hypotheses for further investigation. No formal statistical comparison was conducted. The preliminary conceptual model was reviewed by the clinicians during the interview process to ensure the completeness and accuracy of the developed content.

\section{Patient Interviews}

Semi-structured patient interviews were conducted to elucidate the rpFSGS experience from the patient's perspective. All patients were 
anonymized and provided written consent for inclusion in the study and for the results to be published. To obtain a representative sample of the general rpFSGS population, patients were recruited from a variety of sources, including the National Kidney Foundation patient advocacy group, social media networking sites (Twitter [identified by \#FSGS hashtags and/or FSGS on Google search] and a personal blog), and from clinician referrals from two previously interviewed nephrologists.

For interview participation, patients with rpFSGS were required to meet the following screening criteria: a documented history of pFSGS in their native kidney, received at least one kidney transplant, and had been diagnosed with at least one recurrence of pFSGS. For comparison, kidney transplant recipients with non-recurrent pFSGS and non-kidney transplant patients with pFSGS in their native kidney(s) were also interviewed to discern the patient experience concepts unique to patients with rpFSGS. To be eligible for interview participation, patients with pFSGS and rpFSGS were required to have had pFSGS and be on the kidney transplant waiting list (pre-transplant group), have a history of pFSGS in the native kidney, or to have received at least one kidney transplant without recurrence of pFSGS (posttransplant group) at the time of study. Patients were excluded from the analysis if they had a diagnosis of sFSGS; not undergone a biopsy to confirm a diagnosis of FSGS (verbally confirmed by the patient); current (or a history of) drug addiction, alcohol abuse, or mental disability; an illness rendering them unsuitable for inclusion in the study in the opinion of the investigator; or if they were currently enrolled in a clinical trial. Demographic characteristics (age, gender, education level, and ethnicity) were collected to further characterize the groups but were not used as a means to exclude patients from the study.

To qualitatively examine the differences in the patient experience between the three groups, the predefined set of concepts (identified in the literature review and refined in the clinician interviews) was tested in each group. For patients with rpFSGS, the questions were designed to obtain information about their current symptoms and the impact of rpFSGS on their QoL, as well as to further investigate how patients' current symptoms compare with symptoms at their worst (e.g., before receiving their first kidney transplant). Degree of disturbance on patient QoL was assessed for the different symptoms and impact concepts using patient self-assessment scores (on a scale of 0-10; where 0 indicated that the symptom or impact did not disturb the patient's life at all and 10 indicated that the symptom or impact greatly disturbed the patient's life). For patients with pre-transplant pFSGS, the interview focused on deriving information about current symptoms and the overall health experience relative to their condition. For patients with post-transplant non-recurrent pFSGS, interviews were centered around the presence of current or lingering symptoms and their impact post-transplant, and to explore how these symptoms (or lack of symptoms) compare with the symptoms at their worst (i.e., pre-transplant).

\section{Compliance with Ethics Guidelines}

The study was approved by the New England Independent Review Board, Needham Heights, MA, USA. This study was conducted in accordance with the ethical standards of the New England Independent Review Board and with the 1964 Declaration of Helsinki and its later amendments or comparable ethical standards. Informed consent was obtained from all individual participants included in the study.

\section{RESULTS}

\section{Literature Review and Development of the Preliminary Conceptual Model}

The number of publications retrieved from the literature review is shown in Table 1; of these, 58 sources (review articles $[n=13]$, original research articles $[n=11]$, online patient posts $[n=19]$, articles pertaining to PRO instruments $[n=11]$, and clinical trial results $[n=4]$ ) were relevant to this study and included in the analysis. Overall, very few articles were identified in MEDLINE/PubMed relating to the 
symptoms and impact of rpFSGS compared with pFSGS. In the few articles mentioning the recurrence of pFSGS, recurrence was characterized by the same symptoms as the initial presentation of pFSGS, with little or no reference to any other health experience effects specifically attributed to disease recurrence.

Most published articles concerning "quality of life" outcomes were based on studies pertaining to the event of receiving a kidney transplant, and did not test for rpFSGS (or pFSGS) other than assessing changes in QoL attributed to the actual kidney transplant. As no literature was identified for either pre- or posttransplant non-recurrent patients with pFSGS, chronic kidney disease/ESRD and kidney transplant populations, respectively, were used as proxies for these groups to generate hypotheses surrounding the potential rpFSGS experience.

The literature review findings enabled the development of a preliminary conceptual model of the experience of patients with rpFSGS (Fig. 1). The most frequently reported symptoms in the literature for rpFSGS and/or patients with kidney disease were wide-ranging and included "fatigue/ tiredness", "proteinuria/foamy urine", "loss of strength", "muscle loss", "muscle weakness", and "edema" [13, 16-18]. Symptoms associated with rpFSGS and treatment (i.e., steroids, immunosuppressants, and rituximab use) in the literature, and in the prescribing information for these therapies include "bone/joint pain", "diarrhea", "dizziness", "fatigue/tiredness", and "headaches" [19-23].

On the basis of published literature, patients with rpFSGS and/or the wider transplant population also seem to experience immediate and generalized impact concepts including "anxiety", "decreased social functioning", and "depression" [14, 16, 24, 25]. Impact concepts considered predominantly associated with treatment in patients with kidney disease (but not specific to patients with rpFSGS) included "appetite/weight gain" (associated with corticosteroid use); "decreased satisfaction with appearance" (in patients receiving corticosteroid and immunosuppressant therapy); "nonadherence" and "pill burden" (in patients with poor renal function following transplantation); and "caregiver burden", "withdrawal from dialysis", and "decreased satisfaction with social support network" (in patients receiving dialysis) [21, 26-29].

Online posts from FSGS patients $(n=19)$ confirmed many of the aforementioned symptoms and subsequent effects reported in the literature for rpFSGS and patients with kidney disease; in addition, disease-related factors not reported in the current literature were described in online patient posts and were subsequently incorporated into the rpFSGS conceptual model. These included "frequent urination", "mood swings", and "shakiness" as symptoms, and "financial difficulty", "denial", "extreme disappointment", and "feelings of hopelessness" as impact concepts [17, 30-33]. Impact concepts referenced in online patient forum discussions/ blogs and considered to be both disease- and treatment-related included "aggression/irritableness", "reduced work productivity/inability to work", and "weight/appetite loss" [17, 30-33].

\section{Clinician Interviews and Refinement of the Conceptual Model}

Building on the literature review findings, clinicians perceived the range of symptoms experienced and degree of symptom burden to vary significantly from patient to patient, with some displaying a more aggressive disease course than others. Moreover, the timing of symptom presentation was said to vary among patients with rpFSGS, with some being diagnosed in a delayed fashion during routine post-transplant monitoring. Additionally, dialysis and other treatments, including immunosuppressive therapies, rituximab, and plasmapheresis, were also discussed with clinicians; this highlighted a need to consider the wider impact, including the broader psychological implications of these treatments, on patients with rpFSGS. From the clinician's perspective, suffering a recurrence of rpFSGS was regarded as "devastating" for the patient after having recently undergone transplantation. For this reason, the consequences of the condition were regarded as being potentially magnified beyond that of pFSGS.

On the basis of clinician feedback, three main refinements were made to the preliminary 


\begin{tabular}{|c|c|c|c|}
\hline \multicolumn{2}{|c|}{$\begin{array}{c}\text { Initial treatment } \\
\text { paradigm (steroids) }\end{array}$} & \multicolumn{2}{|c|}{$\begin{array}{l}\text { Patient population: } \\
\text { Patients with rpFSGS }\end{array}$} \\
\hline \multicolumn{4}{|c|}{$\begin{array}{l}\text { Disease process: } \\
\text { - Idiopathic recurrence of localised scarring of the glomeruli post kidney transplantation } \\
\text { Resulting conditions: } \\
\text { - Can progress to ESRD leading to graft failure and potentially death }\end{array}$} \\
\hline \multicolumn{4}{|c|}{ Signs of disease } \\
\hline \multicolumn{2}{|c|}{$\begin{array}{l}\text { - High serum creatinine } \\
\text { - Hyperlipidemia } \\
\text { - Hypertension } \\
\text { - Hypoalbuminemia }\end{array}$} & $\begin{array}{l}\text { - Nephrotic syndrome } \\
\text { - Proteinuria } \\
\text { - Renal insufficiency }\end{array}$ & \\
\hline \multicolumn{4}{|c|}{ Symptoms of disease/treatment } \\
\hline $\begin{array}{l}\text { - Fatigue/tiredness } \\
\text { - Foamy urine } \\
\text { - Loss of strength } \\
\text { - Muscle loss } \\
\text { - Muscle weakness } \\
\text { - Swelling (edema) }\end{array}$ & $\begin{array}{ll}\text { - Chest pain } & \cdot \mathrm{Ha} \\
\text { - Cough } & \cdot \mathrm{Mo} \\
\text { - Dry mouth } & \cdot \mathrm{Mo} \\
\text { - Dryfitchy skin } & \cdot \mathrm{Nig} \\
\text { - Excessive thirst } & \cdot \mathrm{Sh} \\
\text { - Frequent urination } & \cdot \mathrm{Ski} \\
\text { - Muscle cramps } & \cdot \mathrm{Sw} \\
\text { - Muscle fatigue } & \\
\text { - Shortness of breath } & \end{array}$ & $\begin{array}{l}\text { ir growth/loss } \\
\text { od swings } \\
\text { on face (alteration to fac } \\
\text { ht sweats } \\
\text { akiness } \\
\text { in rash } \\
\text { ollen/bleeding gums }\end{array}$ & $\begin{array}{l}\text { - Bone/joint pain } \\
\text { - Diarrhea } \\
\text { - Dizziness } \\
\text { - Headaches } \\
\text { - Nausea } \\
\text { - Stomach pain } \\
\text { - Vomiting }\end{array}$ \\
\hline \multicolumn{4}{|c|}{ Immediate impact } \\
\hline $\begin{array}{l}\text { - Difficulty concentrating } \\
\text { - Sleeplessness }\end{array}$ & $\begin{array}{l}\text { - Aggression/Irritableness } \\
\text { - Decreased satisfaction w } \\
\text { - Financial difficulty } \\
\text { - Pill burden }\end{array}$ & $\begin{array}{ll}\cdot \text { Appe } \\
\text { ith appearance } & \cdot \text { Weic } \\
& \end{array}$ & $\begin{array}{l}\text { - Appetite change (gain/loss) } \\
\text { - Weight change (gain/loss) }\end{array}$ \\
\hline \multicolumn{4}{|c|}{ General impact } \\
\hline $\begin{array}{l}\text { - Anxiety } \\
\text { - Decreased } \\
\text { social functioning } \\
\text { - Depression }\end{array}$ & $\begin{array}{l}\text { - Denial } \\
\text { - Extreme disappointment } \\
\text { - Feelings of hopelessness } \\
\text { - Limitations in daily activities } \\
\text { - Reduced sexual interest } \\
\text { - Stress from lifestyle/diet } \\
\text { changes } \\
\text { - Uncertainty of graft survival }\end{array}$ & $\begin{array}{l}\text { - Caregiver burden } \\
\text { - Nonadherence } \\
\text { - Voluntary withdrawal } \\
\text { from dialysis }\end{array}$ & $\begin{array}{l}\text { - Decreased } \\
\text { satisfaction with } \\
\text { social support } \\
\text { network } \\
\text { - Inability to work/ } \\
\text { be productive at } \\
\text { work }\end{array}$ \\
\hline
\end{tabular}

Fig. 1 Preliminary rpFSGS conceptual model based on findings from the published literature and online patient posts. Bold text: Hypothesized to be the most salient disease-related symptoms/impact concepts. Italicized text: Hypothesized disease-related symptoms/impact concepts.
Blue text: Hypothesized treatment-related symptoms. Green text: Hypothesized disease- and treatment-related symptoms/impact concepts. ESRD end-stage renal disease, rpFSGS recurrent primary focal segmental glomerulosclerosis 
conceptual model of the experience of patients with rpFSGS: (1) the overall range of conditionrelated symptoms was narrowed, on the basis of the clinicians' views that symptoms are not typically as broad as suggested by the literature; (2) a wider range of treatment-related symptoms was introduced, on the basis of the insight that treatment for rpFSGS extends beyond that of steroid use; and (3) the impact related to anxiety and depression was further explored following the consideration that these mental health disorders have broad meanings and implications.

\section{Patient Interviews: Demographics}

In total, 25 patients were recruited from the National Kidney Foundation's patient advocacy groups $(n=17)$, social media networking sites (Twitter [identified by \#FSGS hashtags and/or "FSGS" on Google search]: $n=4$; blog: $n=1$ ), and from clinician referrals from two previously interviewed nephrologists $(n=3)$.

Demographics of interviewed patients with rpFSGS $(n=15)$, pre-transplant pFSGS $(n=5)$, and post-transplant non-recurrent pFSGS $(n=5)$ are shown in Table 2.

\section{Patient Interviews: Symptoms of rpFSGS Graded by Disturbance}

Figure $2 \mathrm{a}$ presents an overview of rpFSGS symptoms, shows how frequently each was reported in the patient interviews, and the average patient-reported degree of disturbance on patient QoL. Additional context is provided with representative patient quotes (Table 3 ). The most salient symptoms were considered to be those that were both frequently reported and rated as being highly disturbing. For patients with rpFSGS, the most salient reported symptoms were tiredness, swelling, shortness of breath, loss of appetite, and dry, itchy skin. "Tiredness" was the most commonly reported symptom, reported by most patients (14/15 mentions) with rpFSGS. Tiredness had a profound negative impact on activities of daily living and represented one of the most disturbing symptoms (Fig. 2a; Table 3). It was further noted as being distinct from "physical weakness".

Swelling (11/15 mentions), also referred to by patients as "edema", "bloating", or "puffiness", was also reported as a common and disturbing symptom of rpFSGS (Fig. 2a; Table 3). In addition, swelling was considered to be one of the few symptoms experienced by patients at the time of diagnosis, and was noted to manifest in different locations of the body, including the feet, calves, legs, stomach, hands, face, around the eyes, and cheeks. Patients also indicated that dialysis helped to alleviate the severity of the swelling. Shortness of breath (10/ 15 mentions), appetite loss (11/15 mentions), and dry or itchy skin (13/15 mentions) were also perceived to be common and disturbing symptoms of rpFSGS (Fig. 2a; Table 3 ).

Overall, 11 out of 15 interviewed patients noted the presence of "foamy or bubbly urine" and/or "decreased urination" as a symptom of rpFSGS, with the former being described in the literature and by clinicians as a hallmark of rpFSGS, resulting from proteinuria [34-36]. Indeed, of the five patients with non-recurrent pFSGS and who were interviewed, none experienced proteinuria post-transplant. All patients with rpFSGS who experienced "foamy or bubbly urine" (9/15 mentions) reported that it became more noticeable as the disease worsened, while patients who experienced "decreased urination" (9/15 mentions), even to the point of absent urination following progression to ESRD, often made the association between the symptom and degree of remaining kidney function (Table 3). Despite the connections made by some patients between the presence of "foamy or bubbly urine" or "decreased urination" and the underlying severity of their condition, patients, on average, rated these symptoms as being relatively low in disturbance (3.0/10 and 3.7/10, respectively; Fig. 2a).

\section{Patient Interviews: Impact Concepts in rpFSGS and Disturbance Ranking}

An overview of all rpFSGS impact concepts, along with their frequency of mention and the average patient-reported degree of disturbance, 
Table 2 Patient interviewee demographics

\begin{tabular}{|c|c|c|c|}
\hline Characteristic & $\begin{array}{l}\text { Post-transplant } \\
\text { rpFSGS }(n=15)^{\mathrm{a}}\end{array}$ & $\begin{array}{l}\text { Pre-transplant } \\
\text { pFSGS }(n=5)\end{array}$ & $\begin{array}{l}\text { Post-transplant non-recurrent } \\
\text { pFSGS }^{b}(n=5)\end{array}$ \\
\hline Median age, years (range) & $45.5(20-57)$ & $49.0(28-73)$ & $39.0(34-46)$ \\
\hline \multicolumn{4}{|l|}{ Gender, $n(\%)$} \\
\hline Female & $13(86.7)$ & $1(20.0)$ & $3(60.0)$ \\
\hline Male & $2(13.3)$ & $4(80.0)$ & $2(40.0)$ \\
\hline \multicolumn{4}{|l|}{ Ethnicity, $n(\%)$} \\
\hline White & $11(73.3)$ & $2(40.0)$ & $2(40.0)$ \\
\hline Black (African American) & $4(26.7)$ & $2(40.0)$ & $1(20.0)$ \\
\hline Asian & - & $1(20.0)$ & $1(20.0)$ \\
\hline Hispanic & - & - & $1(20.0)$ \\
\hline $\begin{array}{l}\text { Previously received one transplant, } \\
n(\%)\end{array}$ & $7(46.7)$ & $\mathrm{N} / \mathrm{A}$ & $4(80.0)$ \\
\hline $\begin{array}{l}\text { Previously received two transplants, } \\
\quad n(\%)\end{array}$ & $8(53.3)$ & $\mathrm{N} / \mathrm{A}$ & $1(20.0)$ \\
\hline $\begin{array}{l}\text { Median time since rpFSGS } \\
\text { diagnosis, years (range) }{ }^{c}\end{array}$ & $3.5(1-17)$ & $\mathrm{N} / \mathrm{A}$ & $\mathrm{N} / \mathrm{A}$ \\
\hline $\begin{array}{l}\text { Median time since pFSGS } \\
\text { diagnosis, years }(\text { range })^{c}\end{array}$ & $15(7-48)$ & $5(2-10)$ & $11(7-19)$ \\
\hline \multicolumn{4}{|c|}{ Disease characteristic (self-reported), $n(\%)$} \\
\hline ESRD & $6(40.0)$ & $5(100.0)$ & $\mathrm{N} / \mathrm{A}$ \\
\hline Declined renal function & $5(33.3)$ & - & \\
\hline Remission & $2(13.3)$ & - & \\
\hline Removed kidneys & $2(13.3)$ & - & \\
\hline
\end{tabular}

ERSD end-stage renal disease, FSGS focal segmental glomerulosclerosis, $N / A$ not applicable, $p F S G S$ primary focal segmental glomerulosclerosis, rpFSGS recurrent primary focal segmental glomerulosclerosis

${ }^{a}$ Median age was not collected for one patient; median time since recurrent FSGS diagnosis was unknown for one patient

b Median age and median time since FSGS diagnosis were not collected for one patient because of an error in recording

c Calculated as March 2017-the patient-reported approximate date of diagnosis during the patient interviews

can be found in Fig. $2 b$, with representative quotes in Table 3. The most salient impact concepts (high frequency and high disturbance rating) were limitations/difficulties performing physical acts, unable to work or be productive at work, less frequent social interactions, anxiety, and frustration. The limitation in physical activity (reported by patients as being mainly due to tiredness or lack of energy) was the most frequently experienced impact of rpFSGS (13/15 mentions), and was also reported to restrict recreational activities (Fig. 2b; Table 3). A decreasing ability to perform these activities because of their condition was a major concern for patients, as reflected by high disturbance rating scores.

Similarly, patients also discussed the significant detrimental effect of rpFSGS on their work 
(a)

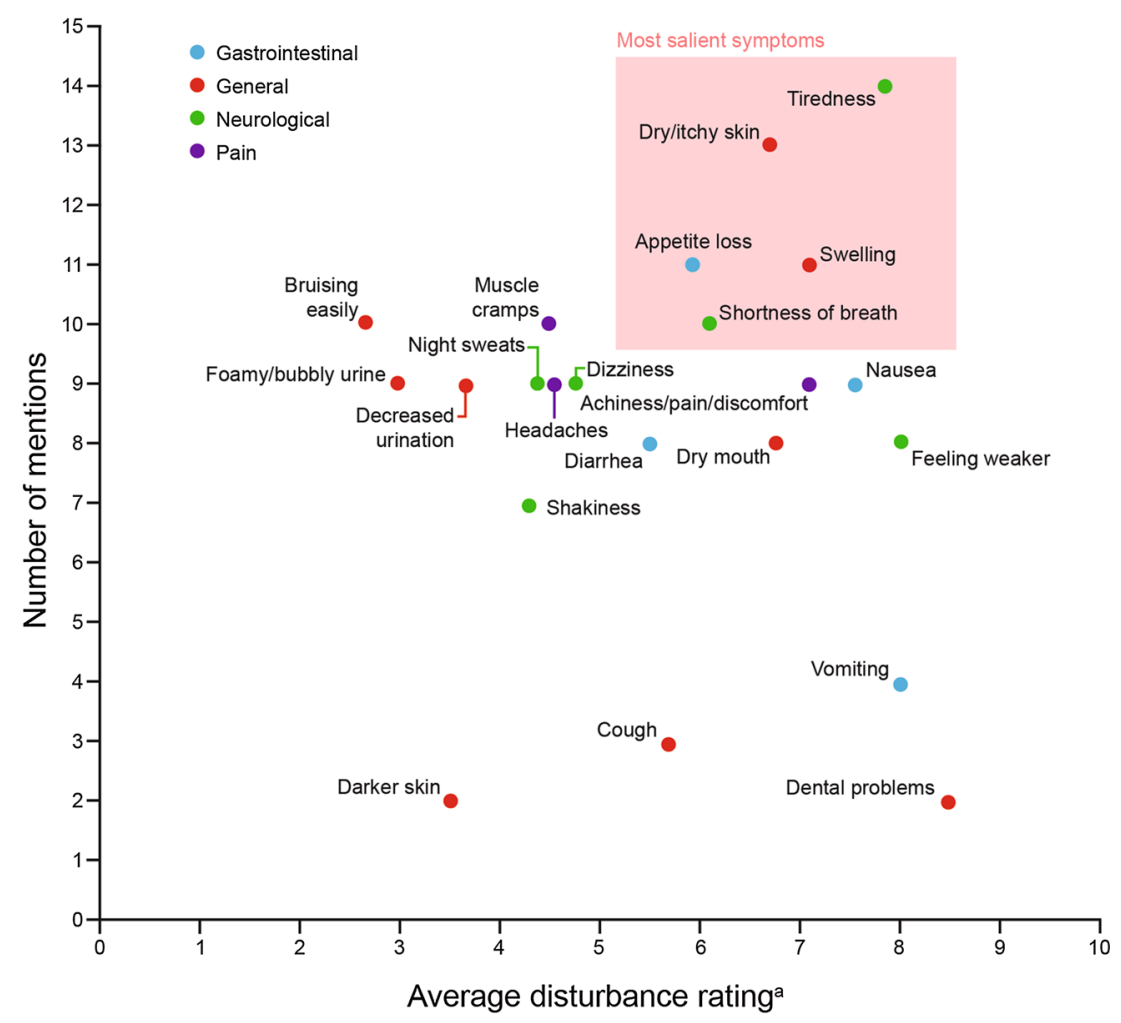

(b)

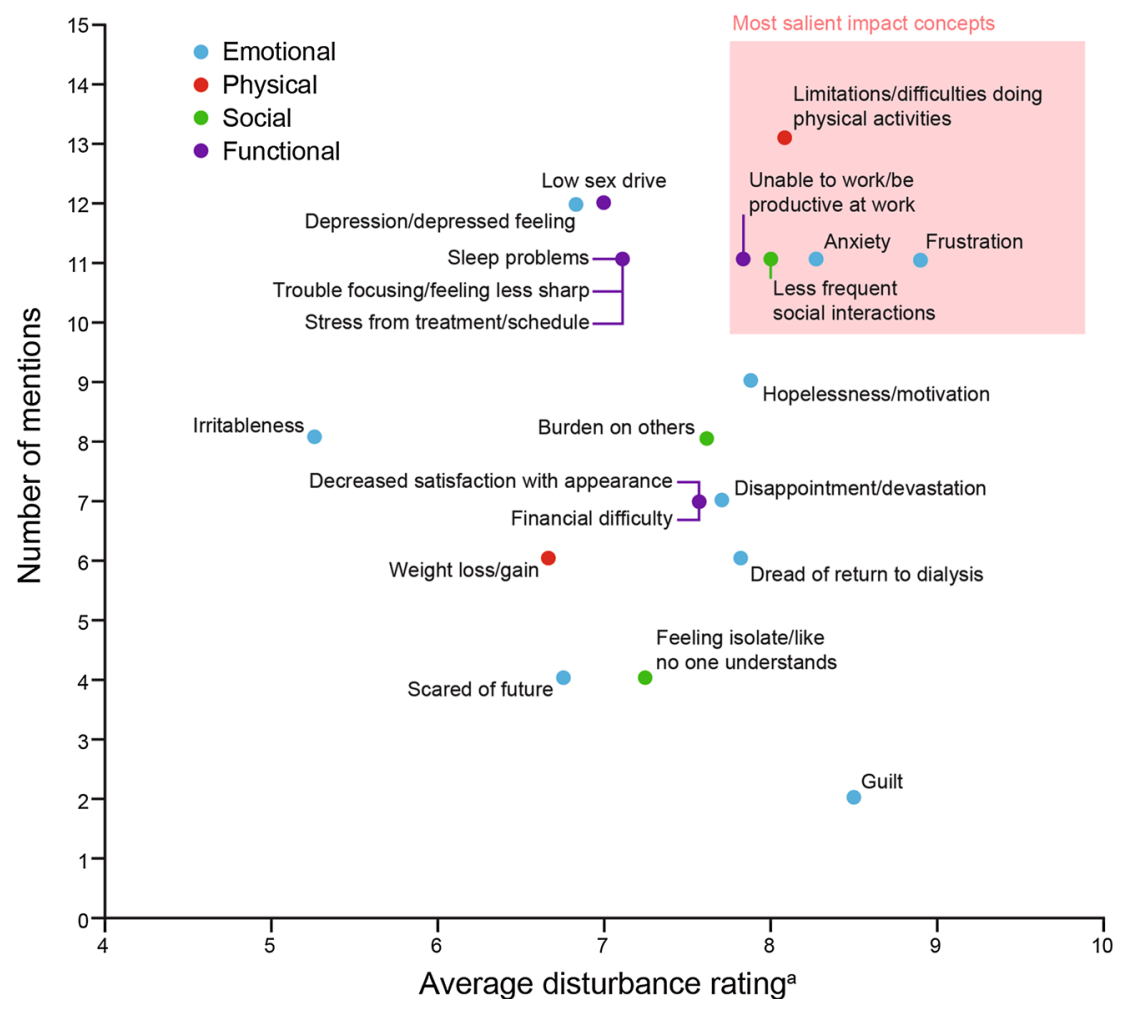


4 Fig. 2 Frequency of symptoms (a) and impact concepts (b) of rpFSGS, and the scale of patient-reported disturbance. ${ }^{\mathrm{a}}$ Disturbance is patient reported and a standard question in the conceptual model development. In this instance, patients were asked for a rating and an explanation as to the rationale for their rating on disturbance. Question: "On a scale of $0-10$, where 0 means that this symptom does not disturb your life at all and a 10 means that this symptom greatly disturbs your life, how much does this symptom disturb your life? Please explain”. rpFSGS recurrent primary focal segmental glomerulosclerosis

productivity and social life (11/15 mentions; Fig. 2b; Table 3). Patients reported experiencing generalized anxiety (11/15 mentions) as a result of needing to manage their rpFSGS and treatment, along with a feeling of uncertainty about their future, regarding this as a fairly disturbing feature of their condition (Fig. 2b; Table 3). Depression or depressed feelings were commonly linked to anxiety in these patients, with approximately half of all interviewees having sought professional help from a therapist or having received psychiatric medication to cope with their feelings of anxiety or depression. In addition to no longer being able to perform the activities they once enjoyed as a result of physical restrictions imposed by their condition, patients also sensed related feelings of frustration (11/15 mentions) associated with an overwhelming sense of powerlessness (Fig. 2b; Table 3).

\section{Patient Interviews: rpFSGS and pFSGS Comparison}

In this study, although patients with rpFSGS and those with pFSGS (pre-transplant) reported the same symptoms and adverse impact (Table 4), the frequency of mention and patient-reported degree of disturbance differed between groups. Patients with rpFSGS, having already undergone a major surgical procedure to overcome their condition, appeared to be more acutely aware of their symptoms and perceived them differently compared with the original pFSGS symptoms they experienced. For example, one patient noted that "I wouldn't say that the symptoms are any different. I'm just more aware of it this time because I actually know what's going on and I'm more tuned into my body". In addition to heightened awareness of the impact of the condition, this finding could also be due to the influence of confounding factors such as age, longer duration of disease, and the influence or benefit of treatment.

As expected, post-transplant patients with non-recurrent pFSGS experienced fewer symptoms compared with those with rpFSGS (Table 4). Although this study identified four symptoms that were common in both groups (diarrhea, dizziness, nausea, and shakiness), the most salient symptoms experienced by patients with rpFSGS were not present in those without recurrence post transplant. As reported in the literature, diarrhea, dizziness, and nausea are considered to be rpFSGS-related and also treatment-related [19-23], suggesting that the lingering symptoms experienced by non-recurrent patients are predominantly treatment-related. The finding that tiredness, swelling, shortness of breath, loss of appetite, and dry, itchy skin were frequently reported and judged as highly disturbing by patients with rpFSGS, but not reported at all by patients with non-recurrent pFSGS, highlights the unmet need among the population of patients with rpFSGS.

Disease impact was limited in the non-recurrent post-transplant recipients (Table 4), particularly in comparison with both the rpFSGS and the pre-transplant pFSGS groups. The only reported impact concepts among patients with post-transplant non-recurrent pFSGS were anxiety, hopelessness/lack of motivation, and stress from treatment schedule. Although anxiety was frequently reported in both the rpFSGS and non-recurrent pFSGS groups, non-recurrent patients notably cited the fear of recurrence as a cause of their anxiety. Similarly, feelings of hopelessness and decreased motivation were documented in both groups but were attributed to a lack of confidence in remaining FSGS-free in the non-recurrent pFSGS group. Both rpFSGS and nonrecurrent rpFSGS groups experienced psychological stress as a result of an intense posttransplant treatment schedule. In the absence 
Table 3 Representative quotations from patients with rpFSGS and non-recurrent pFSGS

\section{Reported symptoms/ Quotations \\ impact}

Symptoms of rpFSGS

Tiredness

"It feels like I have no life-force. It just feels like I have no energy. Like no matter how much sleep I get it's like getting out of bed in the morning is like walking through sand or something. It feels like... I just have no energy no matter what I do"

Swelling

"I had really bad swelling in my legs... by the end of the day my calves would feel so tight. It felt like my skin was like being forced to expand over a ton of fluid and it was very, very painful and tight feeling"

Shortness of breath

"I know at its worst it feels like somebody's got their hands around my throat and I can't breathe. At the worst, it's panicky. It is an absolute panic, especially because it happens when you lay down"

Dry/itchy skin

"The dry skin is terrible. Alligator skin, all the time... when [a rash] happens, it's just extreme itch, all day long, wakes you up in the middle of the night, pretty much all over. It's just everything from the core of your body, some on the back, chest, everywhere”

Appetite loss

"I'm not a big eater. I don't eat a lot of times. It can be 3[pm] and I'll say to myself "Oh, I need to eat.” I eat because it's $3[\mathrm{pm}]$, not because I'm hungry. My appetite isn't real strong”

Foamy/bubbly urine

"It all turned to white foam in the toilet. As the proteinuria got worse, it got foamier. I mean, obviously it's not right. The foamier it gets, it's less right. So, it's like watching things deteriorate"

Decreased urination

"I have less than $2 \%$ [kidney function]... I guess about a year ago I lost the ability to make any urine at all"

Impact of rpFSGS

Physical activity restrictions

'There's things that I used to do, that I can't do any more, like ride my bike. Sometimes I can't even take a walk. That's been really difficult. I feel like I can't do very basic things that most people can do. I have trouble even doing my laundry and things like that"

Work productivity/social "It gives you a feeling of being less than a provider. I like to work. I like to do things to take life care of my family. And you physically just can't do it anymore"

Anxiety/depression

"I dealt with severe depression and anxiety, I didn't know what was going on in my kidney. So emotionally it's hard in the sense that I really can't count on anything. It's all up to my body. I can't do anything without my health. It's just a worry. It's the worry and waiting and anxiety of not knowing, and then knowing, and then just waiting. So many unknowns”

Frustration/sense of powerlessness

“[There's] the frustration of not being able to do, especially physically, everything I wanted to go out and do... it can affect the family, the marriage, and even work, and days I'm literally vomiting, walking out of the house to get to work because I can't be fired, and it gets... it's tough, tough to take” 
Table 3 continued

\begin{tabular}{ll}
$\begin{array}{l}\text { Reported symptoms/ } \\
\text { impact }\end{array}$ & Quotations \\
\hline Disease recurrence & $\begin{array}{l}\text { "It's terrifying. I guess you don't really know what to think. It's just really scary because you're } \\
\text { not really sure what's going to work and what's not going to work. I was worrying that my } \\
\text { life was going to return exactly as it was before the transplant and that I would be on dialysis } \\
\text { forever" }\end{array}$ \\
$\begin{array}{ll}\text { Impact of non-recurrent pFSGS } \\
\text { Absence of disease } \\
\text { recurrence }\end{array}$ & "You don't realize how sick you are until you feel better" \\
& "I felt 100\% better. My color and everything is totally different from prior where I was very \\
puffy. And now, I have energy"
\end{tabular}

$p F S G S$ primary focal segmental glomerulosclerosis, $r p F S G S$ recurrent primary focal segmental glomerulosclerosis

of recurrence, the functional, emotional, and psychological concepts associated with pFSGS were considered to eventually dissipate, with patients resuming a normal QoL. By contrast, patients with rpFSGS recalled a profoundly negative emotional and psychological impact when informed that their disease had recurred (Table 3).

\section{Finalization of the Conceptual Model}

The findings from the literature review, refined via clinician interviews and tested in patient interviews, contributed towards the development of the finalized conceptual model for rpFSGS (Fig. 3).

\section{DISCUSSION}

Importantly, although the majority of patients with rpFSGS (9/15) in our study reported experiencing "foamy/bubbly urine" and reported that it became more noticeable as the disease worsened, they did not rate this symptom as being highly disturbing. Therefore, even though "foamy/bubbly urine" may be a hallmark feature of rpFSGS [34-36], it does not seem to have much impact on patient QoL. Our results showed that the most salient symptoms-those that were reported most frequently and rated as most disturbing-are tiredness, swelling, appetite loss, dry and/or itchy skin, and shortness of breath.

Physical limitations and difficulties meeting the challenges of daily life activities, along with the impact of rpFSGS on patients' professional and social lives, were prevalent in this patient population, and were particularly disturbing for patients. Anxiety and feelings of frustration were commonly reported among patients with rpFSGS, and were inherently associated with a fear of the unknown and the inability to exert control over their condition or future.

In this study, patients with rpFSGS could not be differentiated qualitatively from those with pFSGS in native kidneys on the basis of their symptom/impact profiles alone, with patients with rpFSGS reporting the same symptoms and associated impact as those with pFSGS. Therefore, it appears that rpFSGS may prevent these patients from experiencing symptom relief posttransplant. However, some of the interviewed patients with rpFSGS perceived their current disease experience differently compared with how they felt when the condition was first diagnosed. By contrast, the severity of symptoms and overall impact appear to be markedly different in patients with rpFSGS compared with patients with non-recurrent pFSGS (post transplant). Unlike patients with rpFSGS, patients 
Table 4 Symptoms and impact concepts of post-transplant rpFSGS compared with pre-transplant pFSGS

\begin{tabular}{|c|c|c|c|}
\hline & $\begin{array}{l}\text { Post-transplant } \\
\text { rpFSGS }\end{array}$ & $\begin{array}{l}\text { Pre-transplant } \\
\text { pFSGS }\end{array}$ & $\begin{array}{l}\text { Post-transplant } \\
\text { pFSGS }\end{array}$ \\
\hline \multicolumn{4}{|l|}{ Symptoms } \\
\hline Tiredness $^{\mathrm{a}}$ & $\checkmark$ & $\boldsymbol{V}$ & \\
\hline Dry/itchy skin ${ }^{a}$ & $\checkmark$ & $\boldsymbol{V}$ & \\
\hline Swelling ${ }^{a}$ & $\checkmark$ & $\boldsymbol{V}$ & \\
\hline Appetite loss ${ }^{\mathrm{a}}$ & $\boldsymbol{V}$ & $\boldsymbol{V}$ & \\
\hline Shortness of breath ${ }^{a}$ & $\checkmark$ & $\boldsymbol{V}$ & \\
\hline Achiness/pain/discomfort ${ }^{\mathrm{b}}$ & $\checkmark$ & $\boldsymbol{V}$ & \\
\hline Cough $^{\text {b }}$ & $\checkmark$ & $\boldsymbol{V}$ & \\
\hline Darker skin ${ }^{\mathrm{b}}$ & $\checkmark$ & $\boldsymbol{V}$ & \\
\hline Decreased urination ${ }^{\mathrm{b}}$ & $\checkmark$ & $\checkmark$ & \\
\hline Dental problems ${ }^{\mathrm{b}}$ & $\checkmark$ & $\boldsymbol{\sim}$ & \\
\hline Feeling weak ${ }^{\mathrm{b}}$ & $\checkmark$ & $\checkmark$ & \\
\hline Foamy/bubbly urine ${ }^{\mathrm{b}}$ & $\checkmark$ & $\checkmark$ & \\
\hline Headaches ${ }^{\mathrm{b}}$ & $\checkmark$ & $\checkmark$ & \\
\hline Bruising easily $^{c}$ & $\checkmark$ & $\boldsymbol{V}$ & \\
\hline Diarrhea $^{c}$ & $\checkmark$ & $\boldsymbol{V}$ & $\checkmark$ \\
\hline Dizziness $^{c}$ & $\checkmark$ & $\checkmark$ & $\checkmark$ \\
\hline Dry mouth ${ }^{c}$ & $\checkmark$ & $\checkmark$ & \\
\hline Muscle cramps ${ }^{c}$ & $\checkmark$ & $\checkmark$ & \\
\hline Nausea $^{c}$ & $\checkmark$ & $\checkmark$ & $\checkmark$ \\
\hline Night sweats ${ }^{c}$ & $\checkmark$ & $\checkmark$ & \\
\hline Shakiness ${ }^{c}$ & $\boldsymbol{\nu}$ & $\boldsymbol{V}$ & $\checkmark$ \\
\hline Vomiting ${ }^{c}$ & $\checkmark$ & $\checkmark$ & \\
\hline \multicolumn{4}{|l|}{ Immediate impact } \\
\hline $\begin{array}{l}\text { Limitations/difficulties doing physical } \\
\text { activities }^{\mathrm{a}}\end{array}$ & $\checkmark$ & $\boldsymbol{V}$ & \\
\hline Less frequent social interactions ${ }^{a}$ & $\checkmark$ & $\checkmark$ & \\
\hline Unable to work/be productive at work ${ }^{a}$ & $\checkmark$ & $\boldsymbol{V}$ & \\
\hline Disappointment/devastation of outcome ${ }^{\mathrm{b}}$ & $\checkmark$ & $\checkmark$ & \\
\hline Pain from skin stretching ${ }^{\mathrm{b}}$ & $\checkmark$ & $\checkmark$ & \\
\hline Scared of future ${ }^{b}$ & $\checkmark$ & $\checkmark$ & \\
\hline Sleep problems ${ }^{\mathrm{b}}$ & $\checkmark$ & $\checkmark$ & \\
\hline
\end{tabular}


Table 4 continued

\begin{tabular}{|c|c|c|c|}
\hline & $\begin{array}{l}\text { Post-transplant } \\
\text { rpFSGS }\end{array}$ & $\begin{array}{l}\text { Pre-transplant } \\
\text { pFSGS }\end{array}$ & $\begin{array}{l}\text { Post-transplant } \\
\text { pFSGS }\end{array}$ \\
\hline Trouble focusing/feeling less sharp ${ }^{\mathrm{b}}$ & $\checkmark$ & $\checkmark$ & \\
\hline Decreased satisfaction with appearance $^{c}$ & $\checkmark$ & $\checkmark$ & \\
\hline Dread of return to dialysis ${ }^{c}$ & $\checkmark$ & $\checkmark$ & \\
\hline Irritableness $^{c}$ & $\checkmark$ & $\boldsymbol{V}$ & \\
\hline Weight loss/gain ${ }^{c}$ & $\checkmark$ & $\boldsymbol{V}$ & \\
\hline \multicolumn{4}{|l|}{ General impact } \\
\hline Frustration $^{a}$ & $\checkmark$ & $\checkmark$ & \\
\hline Anxiety ${ }^{a}$ & $\checkmark$ & $\checkmark$ & $\checkmark$ \\
\hline Burden on others ${ }^{b}$ & $\checkmark$ & $\boldsymbol{V}$ & \\
\hline Depression/depressed feeling ${ }^{\mathrm{b}}$ & $\checkmark$ & $\checkmark$ & \\
\hline Feeling isolated/like no one understands & $\checkmark$ & $\checkmark$ & \\
\hline Financial difficulty ${ }^{\mathrm{b}}$ & $\checkmark$ & $\checkmark$ & \\
\hline Frustration $^{\mathrm{b}}$ & $\checkmark$ & $\checkmark$ & \\
\hline Guilt $^{\mathrm{b}}$ & $\checkmark$ & $\checkmark$ & \\
\hline Hopelessness/motivation ${ }^{\mathrm{b}}$ & $\checkmark$ & $\checkmark$ & $\boldsymbol{\sim}$ \\
\hline Low sex drive ${ }^{\mathrm{b}}$ & $\checkmark$ & $\checkmark$ & \\
\hline Stress from treatment/schedule ${ }^{c}$ & $\checkmark$ & $\checkmark$ & \\
\hline
\end{tabular}

$p F S G S$ primary focal segmental glomerulosclerosis, $r p F S G S$ recurrent primary focal segmental glomerulosclerosis

a Symptoms/impact concepts shown in bold were considered the most salient disease-related (i.e., the most commonly mentioned and most disturbing to patients)

b Symptoms/impact concepts considered disease-related, as reported by patients

c Symptoms/impact concepts considered both disease- and treatment-related, as reported by patients

who were FSGS-free became almost asymptomatic post transplant, had only a few lingering psychological manifestations (anxiety or fear of recurrence, and stress linked to posttransplant treatment), and were largely able to resume a normal life.

There were several limitations associated with the patient interviews conducted in this analysis, and inherent biases may have skewed the results. Selection bias may have occurred, as the patients interviewed were members of a patient advocacy organization; given their engagement in active disease management through advocacy participation, they may have been more attuned to signs, symptoms, and impact associated with rpFSGS. Likewise, such patients may have been more open about their condition compared with the general rpFSGS population. Similarly, patient age and disease duration were also factors that may have influenced patient response. For example, older patients may associate certain symptoms (e.g., tiredness, physical limitations/difficulties, and decreased libido) with age, and may therefore find them less disturbing than younger patients. Patients who have had the disease for a longer duration may also be more accustomed to their illness and may be more (or less) sensitive to 


\begin{tabular}{|c|c|c|c|c|c|c|}
\hline \multicolumn{3}{|c|}{ Treatment ${ }^{\mathrm{a}}$ action } & \multicolumn{4}{|c|}{$\begin{array}{l}\text { Patient population: } \\
\text { Patients with rpFSGS }\end{array}$} \\
\hline \multicolumn{7}{|c|}{$\begin{array}{l}\text { Disease process: } \\
\text { - Idiopathic recurrence of localised scarring of the glomeruli post kidney transplantation } \\
\text { Resulting conditions: } \\
\text { - Can progress to ESRD leading to graft failure and potentially death }\end{array}$} \\
\hline \multicolumn{7}{|c|}{ Signs of disease } \\
\hline \multicolumn{7}{|c|}{$\begin{array}{l}\text { - Anemia } \\
\text { - High serum creatinine } \\
\text { - Hyperlipidemia } \\
\text { - Hypertension }\end{array}$} \\
\hline \multicolumn{7}{|c|}{ Symptoms of disease/treatment } \\
\hline \multicolumn{3}{|c|}{$\begin{array}{ll}\text { - Tiredness (exhaustion, } & \cdot \text { Achiness/pain/discomfort } \\
\text { fatigue, lack of energy) } & \cdot \text { Cough } \\
\text { - Dry/itchy skin } & \cdot \text { Darker skin } \\
\text { - Swelling } & \text { - Dental problems } \\
\text { - Appetite loss } & \text { - Feeling weak } \\
\text { - Shortness of breath } & \cdot \text { Foamy/bubbly urine } \\
& \cdot \text { Headaches }\end{array}$} & \multicolumn{2}{|c|}{$\begin{array}{l}\text { - Bruising easily } \\
\text { - Decreased urination } \\
\text { - Diarrhea } \\
\text { - Dizziness } \\
\text { - Dry mouth }\end{array}$} & $\begin{array}{l}\text { - Mus } \\
\text { - Naus } \\
\text { - Nigh } \\
\text { - Shak } \\
\text { - Vom }\end{array}$ & $\begin{array}{l}\text { cramps } \\
\text { weats } \\
\text { ess }\end{array}$ \\
\hline \multicolumn{7}{|c|}{ Immediate impact } \\
\hline \multicolumn{2}{|c|}{$\begin{array}{l}\text { - Limitations/difficulties } \\
\text { doing physical } \\
\text { activities (recreational } \\
\text { and household) } \\
\text { - Less frequent social } \\
\text { interaction } \\
\text { - Unable to work/be } \\
\text { productive at work }\end{array}$} & \multicolumn{2}{|c|}{$\begin{array}{l}\text { - Disappointment/devastation of outcome } \\
\text { - Scared of future } \\
\text { - Sleep problems } \\
\text { - Trouble focusing/feeling less sharp }\end{array}$} & \multicolumn{3}{|c|}{$\begin{array}{l}\text { - Decreased satisfaction } \\
\text { with appearance } \\
\text { - Dread of return to dialysis } \\
\text { - Irritableness } \\
\text { - Weight loss/gain }\end{array}$} \\
\hline \multicolumn{7}{|c|}{ General impact } \\
\hline $\begin{array}{l}\text { - Frustration } \\
\text { - Anxiety }\end{array}$ & \multicolumn{4}{|c|}{$\begin{array}{l}\text { - Burden on others } \\
\text { - Depression/depressed feeling } \\
\text { - Feeling isolated/like no one understands } \\
\text { - Financial difficulty } \\
\text { - Guilt } \\
\text { - Hopelessness/motivation } \\
\text { - Low sex drive }\end{array}$} & treatm & /schedule \\
\hline
\end{tabular}

Fig. 3 The final rpFSGS conceptual model. Based on qualitative interviews with patients with rpFSGS $(n=15)$. Bold text: Most salient ${ }^{\mathrm{b}}$ disease-related symptoms/impact concepts. Italicized text: Disease-related symptoms/impact concepts. Green text: Disease- and treatment-related

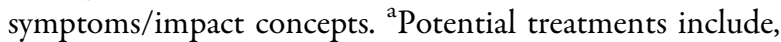
but are not limited to, plasmapheresis, rituximab, immunosuppressants, steroids, angiotensin-converting enzyme inhibitors, angiotensin II receptor blockers, calcineurin inhibitors, and dialysis; ${ }^{b}$ Most salient concepts are both the most mentioned and disturbing to patients. ESRD end-stage renal disease, rpFSGS recurrent primary focal segmental glomerulosclerosis 
disease- and/or treatment-related impact. Owing to the aforementioned factors and the relatively small sample sizes used in this analysis, it was not possible to fully differentiate the symptom/impact profile of patients with rpFSGS from patients with pre-transplant pFSGS and post-transplant non-recurrent pFSGS. However, on the basis of these findings, we identified some important differences that provide scope for further research, including the possibility of future PRO instrument development for use in populations of patients with FSGS.

\section{CONCLUSION}

Post-transplant recipients with rpFSGS display an overall greater symptom burden and experience a wider range of impact concepts compared with patients without disease recurrence, highlighting the unmet need in patients with rpFSGS. We observed that the symptoms and overall impact of rpFSGS largely resemble those observed in patients with pFSGS in their native kidney. Overall, the results of this study emphasize the importance of monitoring patients for recurrence after transplant surgery and developing effective treatments to prevent and manage recurrence in the FSGS setting.

\section{ACKNOWLEDGEMENTS}

The authors would like to thank the participants of the study and Karen Kariv for her valuable contributions to the study and to the development of this manuscript.

Funding. This study was funded by Astellas Pharma Global Development, Inc. The Rapid Service and Open Access Fees were also funded by Astellas Pharma Global Development, Inc.

Medical Writing Assistance. Medical writing support in the development of this manuscript was provided by Robyn Fowler and Matthew Reynolds from OPEN Health Medical Communications (London, UK), funded by Astellas Pharma Global Development, Inc.
Authorship. All named authors meet the International Committee of Medical Journal Editors (ICMJE) criteria for authorship for this article, take responsibility for the integrity of the work as a whole, and have given their approval for this version to be published.

Disclosures. Marci English is an employee of employees of Astellas Pharma Global Development, Inc. Mysore S. A. Kumar is an employee of Astellas Pharma Global Development, Inc. Jason Schwartz is an employee of Astellas Pharma Global Development, Inc. Emily Hawryluk is an employee of IQVIA, who was contracted by Astellas Pharma Global Development, Inc. to provide support for this study. Robert Krupnick is an employee of IQVIA, who was contracted by Astellas Pharma Global Development, Inc. to provide support for this study.

Compliance with Ethics Guidelines. The study was approved by the New England Independent Review Board, Needham Heights, MA, USA. This study was conducted in accordance with the ethical standards of the New England Independent Review Board and with the 1964 Declaration of Helsinki and its later amendments or comparable ethical standards. Informed consent was obtained from all individual participants included in the study.

Data Availability. Access to anonymized individual participant level data will not be provided for this trial because it meets one or more of the exceptions described on http:// www.clinicalstudydatarequest.com under "Sponsor Specific Details for Astellas".

Open Access. This article is distributed under the terms of the Creative Commons Attribution-NonCommercial 4.0 International License (http://creativecommons.org/licenses/ by-nc/4.0/), which permits any noncommercial use, distribution, and reproduction in any medium, provided you give appropriate credit to the original author(s) and the source, provide a link to the Creative Commons license, and indicate if changes were made. 


\section{REFERENCES}

1. Kim JS, Han BG, Choi SO, Cha SK. Secondary focal segmental glomerulosclerosis: from podocyte injury to glomerulosclerosis. Biomed Res Int. 2016;2016:1630365.

2. Fogo AB. Causes and pathogenesis of focal segmental glomerulosclerosis. Nat Rev Nephrol. 2015;11:76-87.

3. D'Agati VD, Kaskel FJ, Falk RJ. Focal segmental glomerulosclerosis. $\mathrm{N}$ Engl J Med. 2011;365:2398-411.

4. Cosio FG, Cattran DC. Recent advances in our understanding of recurrent primary glomerulonephritis after kidney transplantation. Kidney Int. 2017;91:304-14.

5. Rosenberg AZ, Kopp JB. Focal segmental glomerulosclerosis. Clin J Am Soc Nephrol. 2017;12:502-17.

6. McGrogan A, Franssen CF, de Vries CS. The incidence of primary glomerulonephritis worldwide: a systematic review of the literature. Nephrol Dial Transplant. 2011;26:414-30.

7. Sim JJ, Batech M, Hever A, et al. Distribution of biopsy-proven presumed primary glomerulonephropathies in 2000-2011 among a racially and ethnically diverse US population. Am J Kidney Dis. 2016;68:533-44.

8. Messina M, Gallo E, Mella A, Pagani F, Biancone L. Update on the treatment of focal segmental glomerulosclerosis in renal transplantation. World J Transplant. 2016;6:54-68.

9. Kowalewska J. Pathology of recurrent diseases in kidney allografts: membranous nephropathy and focal segmental glomerulosclerosis. Curr Opin Organ Transplant. 2013;18:313-8.

10. Beaudreuil S, Lorenzo HK, Elias M, Nnang Obada E, Charpentier B, Durrbach A. Optimal management of primary focal segmental glomerulosclerosis in adults. Int J Nephrol Renovasc Dis. 2017;10:97-107.

11. Staeck O, Halleck F, Budde K, Khadzhynov D. Longterm outcomes of kidney transplant recipients with primary idiopathic focal segmental glomerulosclerosis. Transplant Proc. 2017;49:2256-9.

12. Kidney Disease Improving Global Outcomes Glomerulonephritis Working Group. KDIGO clinical practice guideline for glomerulonephritis. Kidney Int Suppl. 2012;2:259-74.

13. Gipson DS, Trachtman H, Kaskel FJ, et al. Clinical trials treating focal segmental glomerulosclerosis should measure patient quality of life. Kidney Int. 2011;79:678-85.

14. Liborio AB, Santos JP, Minete NF, et al. Proteinuria is associated with quality of life and depression in adults with primary glomerulopathy and preserved renal function. PLoS One. 2012;7:e37763.

15. Mathias SD, Vallow S, Gipson DS, Thorneloe KS, Sprecher D. Development of focal segmental glomerulosclerosis patient-reported outcome measures: symptom diary and symptom impact questionnaire. Am J Kidney Dis. 2017;70:532-40.

16. Abdel-Kader K, Unruh ML, Weisbord SD. Symptom burden, depression, and quality of life in chronic and end-stage kidney disease. Clin J Am Soc Nephrol. 2009;4:1057-64.

17. NephSpace-NephCure Kidney International's online support community (online patient forum). Patient stories. 2016. http://nephcure.org/connect/ nephspace/. Accessed Oct 2016.

18. Hays RD, Kallich JD, Mapes DL, Coons SJ, Carter WB. Development of the kidney disease quality of life (KDQOL) instrument. Qual Life Res. 1994;3:329-38.

19. Bose B, Cattran D, Toronto Glomerulonephritis Registry. Glomerular diseases: FSGS. Clin J Am Soc Nephrol. 2014;9:626-32.

20. Braun N, Schmutzler F, Lange $\mathrm{C}$ et al. Immunosuppressive treatment for focal segmental glomerulosclerosis in adults. Cochrane Database Syst Rev. 2008:CD003233.

21. Franke GH, Reimer J, Kohnle M, Luetkes P, Maehner $\mathrm{N}$, Heemann U. Quality of life in end-stage renal disease patients after successful kidney transplantation: development of the ESRD symptom checklist - transplantation module. Nephron. 1999;83:31-9.

22. Horizon Pharma USA Inc. Prednisone (RAYOS) Prescribing Information. 2012. Accessed Dec 2018.

23. Novartis Pharmaceuticals Corporation. Cyclosporine (Neoral) Prescribing Information. 2015. Accessed Dec 2018. 2015.

24. Molnar-Varga M, Molnar MZ, Szeifert L, et al. Health-related quality of life and clinical outcomes in kidney transplant recipients. Am J Kidney Dis. 2011;58:444-52.

25. von der Lippe N, Waldum B, Osthus TB, Reisaeter $\mathrm{AV}$, Os I. Health related quality of life in patients in dialysis after renal graft loss and effect of gender. BMC Womens Health. 2014;14:34. 
26. Fiebiger W, Mitterbauer C, Oberbauer R. Healthrelated quality of life outcomes after kidney transplantation. Health Qual Life Outcomes. 2004;2:2.

27. Hardinger KL, Hutcherson T, Preston D, Murillo D. Influence of pill burden and drug cost on renal function after transplantation. Pharmacotherapy. 2012;32:427-32.

28. Mohr PE, Neumann PJ, Franco SJ, Marainen J, Lockridge R, Ting G. The case for daily dialysis: its impact on costs and quality of life. Am J Kidney Dis. 2001;37:777-89.

29. Leggat JE Jr, Bloembergen WE, Levine G, HulbertShearon TE, Port FK. An analysis of risk factors for withdrawal from dialysis before death. J Am Soc Nephrol. 1997;8:1755-63.

30. Nephrotic syndrome and FSGS support group and discussion community (online patient forum). Nephrotic syndrome and FSGS. 2016. https://www. inspire.com/groups/nephrotic-syndrome-and-fsgs/. Accessed Oct 2016.
31. Patient blog. FSGS sucks. 2016. http://fsgssucks. blogspot.com/. Accessed Oct 2016.

32. Patient blog. My kidneys hate me. 2016. http:// mykidneyshatemeblog.blogspot.com/. Accessed Oct 2016.

33. Patient blog. The healing road: FSGS. 2016. http:// thehealingroadfsgs.blogspot.com/. Accessed Oct 2016.

34. Dantas M, Barros Silva GE, Moyses-Neto M. Foamy urine in nephrotic syndrome. Clin Kidney J. 2013;6:341.

35. Kang KK, Choi JR, Song JY, et al. Clinical significance of subjective foamy urine. Chonnam Med J. 2012;48:164-8.

36. Pal A, Kaskel F. History of nephrotic syndrome and evolution of its treatment. Front Pediatr. 2016;4:56. 